\title{
Analysis of Subgrid Stabilization Method for Stokes-Darcy Problems
}

\author{
Kamel Nafa \\ Department of Mathematics and Statistics, Sultan Qaboos University, College of Science, P.O. Box 36, \\ Al-Khoudh 123, Muscat, Oman \\ Correspondence should be addressed to Kamel Nafa; nkamel@squ.edu.om
}

Received 27 April 2016; Revised 31 July 2016; Accepted 16 August 2016

Academic Editor: Weimin Han

Copyright (C) 2016 Kamel Nafa. This is an open access article distributed under the Creative Commons Attribution License, which permits unrestricted use, distribution, and reproduction in any medium, provided the original work is properly cited.

A number of techniques, used as remedy to the instability of the Galerkin finite element formulation for Stokes like problems, are found in the literature. In this work we consider a coupled Stokes-Darcy problem, where in one part of the domain the fluid motion is described by Stokes equations and for the other part the fluid is in a porous medium and described by Darcy law and the conservation of mass. Such systems can be discretized by heterogeneous mixed finite elements in the two parts. A better method, from a computational point of view, consists in using a unified approach on both subdomains. Here, the coupled Stokes-Darcy problem is analyzed using equal-order velocity and pressure approximation combined with subgrid stabilization. We prove that the obtained finite element solution is stable and converges to the classical solution with optimal rates for both velocity and pressure.

\section{Introduction}

The transport of substances between surface water and groundwater has attracted a lot of interest into the coupling of viscous flows and porous media flows [1-5]. In this work we consider coupled problems in fluid dynamics where the fluid in one part of the domain is described by the Stokes equations and in the other, porous media part, by the Darcy equation and mass conservation. Velocity and pressure on these two parts are mutually coupled by interface conditions derived in [6]. Such systems can be discretized by heterogeneous finite elements as analyzed by Layton et al. [1]. In more recent works, unified approaches become more popular. For instance, discontinuous Galerkin methods were analyzed by Girault and Rivière [3], mixed methods by Karper et al. [4], and local pressure gradient stabilized methods by Braack and Nafa [7].

In this work, we consider the $L^{2}$-formulation of the coupled Stokes-Darcy problem as in [4], but we discretize by equal-order finite elements and use subgrid method and grad-div term to stabilize the pressure and control the natural $H^{1}$ (div) velocity norm on the Darcy subdomain.

\section{Formulations of the Stokes-Darcy Coupled Equations}

2.1. Model Equations. Let $\Omega \subset R^{d}, d=2$ or 3 , be a bounded region with Lipschitz boundary $\partial \Omega . \Omega_{S}$ and $\Omega_{D}$ are, respectively, the fluid and porous media subdomains of $\Omega$ such that $\Omega_{S} \cap \Omega_{D}=\emptyset$. The subdomains have a common interface $\Gamma=\bar{\Omega}_{S} \cap \bar{\Omega}_{D}$. We denote by $\mathbf{v}=\left(\mathbf{v}_{S}, \mathbf{v}_{D}\right)$ the fluid velocity and by $p=\left(p_{S}, p_{D}\right)$ the fluid pressure, where $\mathbf{v}_{i}=\left.\mathbf{v}\right|_{\Omega_{i}}, p_{i}=\left.p\right|_{\Omega_{i}}, i=S, D$. The flow in the domain $\Omega_{S}$ is assumed to be of Stokes type and governed by the equations

$$
\begin{aligned}
-2 v \operatorname{div}\left(D\left(\mathbf{v}_{S}\right)\right)+\nabla p_{S}=\mathbf{f}, & & \text { in } \Omega_{S} \\
\operatorname{div} \mathbf{v}_{S}=0, & & \text { in } \Omega_{S}
\end{aligned}
$$

with symmetric strain tensor $D\left(\mathbf{v}_{S}\right)=(1 / 2)\left(\nabla \mathbf{v}_{S}+\nabla \mathbf{v}_{S}^{T}\right)$, external force $\mathbf{f}$, and constant viscosity $v>0$. In the porous region $\Omega_{D}$ the filtration of an incompressible flow through porous media is described by Darcy equations

$$
\begin{aligned}
K^{-1} \mathbf{v}_{D}+\nabla p_{D}=\mathbf{f}, & \text { in } \Omega_{D} \\
\operatorname{div} \mathbf{v}_{D}=g, & \text { in } \Omega_{D},
\end{aligned}
$$


where the permeability $K=K(x)$ is a positive definite symmetric tensor and $g$ denotes an external Darcy force.

2.2. Boundary Conditions. On $\Gamma_{S}=\partial \Omega_{S} \backslash \Gamma$, we prescribe homogeneous Dirichlet conditions for the velocity $\mathbf{v}_{S}$.

$$
\mathbf{v}_{S}=\mathbf{0}, \quad \text { on } \Gamma_{S} .
$$

The boundary of $\Omega_{D}$ is split into three parts $\partial \Omega_{D}=\Gamma \mathrm{U}$ $\Gamma_{D, 1} \cup \Gamma_{D, 2}$. We prescribe zero flux on $\Gamma_{D, 1}$ and a homogeneous Dirichlet condition for the pressure on $\Gamma_{D, 2}$.

$$
\begin{aligned}
& \mathbf{v}_{D} \cdot \mathbf{n}_{D}=0, \quad \text { on } \Gamma_{D, 1} \\
& p_{D}=0, \quad \text { on } \Gamma_{D, 2},
\end{aligned}
$$

where $\mathbf{n}_{D}$ denotes the outer normal vector on the boundary pointing from $\Omega_{D}$ into $\Omega_{S}$. This boundary condition ensures a zero mass flux.

2.3. The Beavers-Joseph-Saffman Condition. The flows in $\Omega_{S}$ and $\Omega_{D}$ are coupled across the interface $\Gamma$. Conditions describing the interaction of the flows are as follows $[6,8]$ :

(i) The continuity of the normal velocity:

$$
\mathbf{v}_{S} \cdot \mathbf{n}_{S}=-\mathbf{v}_{D} \cdot \mathbf{n}_{D}, \quad \text { on } \Gamma
$$

(ii) The balance of normal forces:

$$
-\left(-p_{S} I+2 \nu D\left(\mathbf{v}_{S}\right)\right) \mathbf{n}_{S} \cdot \mathbf{n}_{S}=p_{D}, \quad \text { on } \Gamma
$$

(iii) The Beavers-Joseph-Saffman condition written in terms of the strain tensor:

$$
\mathbf{v}_{S} \cdot \tau=-\frac{2 \sqrt{\widetilde{k}}}{\alpha}\left(D\left(\mathbf{v}_{S}\right) \cdot \mathbf{n}_{S}\right) \cdot \tau,
$$

where $\widetilde{k}=\nu K \tau \cdot \tau$ and $\alpha$ is a dimensionless parameter to be determined experimentally, this condition relating the tangential slip velocity $\mathbf{v}_{S} \cdot \tau$ to the normal derivative of the tangential velocity component in the Stokes region

\section{Variational Formulation}

As variational formulation we consider the so-called $L^{2}$ formulation used by Karper et al. [4] and recently by $[9,10]$. We denote

$$
\begin{aligned}
& (\mathbf{v}, \mathbf{w})_{\Omega}=\int_{\Omega} \mathbf{v w} d x, \quad \mathbf{v}, \mathbf{w} \in L^{2}(\Omega)^{d}, \\
& \langle v, w\rangle_{\Gamma}=\int_{\Gamma} v w d s, \quad v, w \in L^{2}(\Gamma),
\end{aligned}
$$

where $L^{2}(\Omega)$ and $H^{1}(\Omega)$ denote the usual Sobolev spaces.

Next, we define the spaces

$$
\begin{aligned}
& \mathbf{H}_{\Gamma_{S}}^{1}\left(\Omega_{S}\right)=\left\{\mathbf{w} \in\left(H^{1}\left(\Omega_{S}\right)\right)^{d} \mid \mathbf{w}=\mathbf{0} \text { on } \Gamma_{S}\right\} \\
& \mathbf{H}^{1}\left(\operatorname{div}, \Omega_{D}\right)=\left\{\mathbf{w} \in L^{2}\left(\Omega_{D}\right)^{d} \mid \operatorname{div} \mathbf{w} \in L^{2}\left(\Omega_{D}\right)\right\}, \\
& \mathbf{H}_{\Gamma_{D, 1}}^{1}\left(\Omega_{D}\right)=\left\{\mathbf{w} \in \mathbf{H}^{1}\left(\operatorname{div}, \Omega_{D}\right) \mid \mathbf{w} \cdot \mathbf{n}_{D}=0 \text { on } \Gamma_{D}\right\} .
\end{aligned}
$$

Then, multiplying the Stokes equations (1) by the test functions $\mathbf{w}_{S} \in \mathbf{H}_{\Gamma_{S}}^{1}\left(\Omega_{S}\right), q_{S} \in L^{2}\left(\Omega_{S}\right)$, respectively, and integrating by part on the domain $\Omega_{S}$, we obtain

$$
\begin{aligned}
& \left(2 \nu D\left(\mathbf{v}_{S}\right), D\left(\mathbf{w}_{S}\right)\right)_{\Omega_{S}}-\left\langle 2 \nu D\left(\mathbf{v}_{S}\right) \mathbf{n}_{S}, \mathbf{w}_{S}\right\rangle \\
& \quad-\left(p_{S}, \operatorname{div} \mathbf{w}_{S}\right)_{\Omega_{S}}+\left\langle p_{S}, \mathbf{w}_{S} \cdot \mathbf{n}_{S}\right\rangle_{\Gamma}=\left(\mathbf{f}, \mathbf{w}_{S}\right)_{\Omega_{S}}, \\
& \left(\operatorname{div} \mathbf{v}_{S}, q_{S}\right)_{\Omega_{S}}=0 .
\end{aligned}
$$

Using the decomposition $\mathbf{w}_{S}=\left(\mathbf{w}_{S} \cdot \mathbf{n}_{S}\right) \mathbf{n}_{S}+\left(\mathbf{w}_{S} \cdot \tau\right) \tau$, the fluid normal stress condition (6), and the BJS interface condition (7) in (10), we obtain the weak formulation of the Stokes equations: find $\mathbf{v}_{S} \in \mathbf{H}_{\Gamma_{S}}^{1}\left(\Omega_{S}\right), p_{S} \in L^{2}\left(\Omega_{S}\right)$ such that

$$
\begin{aligned}
& \left(2 \nu D\left(\mathbf{v}_{S}\right), D\left(\mathbf{w}_{S}\right)\right)_{\Omega_{S}}+\frac{\nu \alpha}{\sqrt{\tilde{k}}}\left\langle\mathbf{v}_{S} \cdot \tau, \mathbf{w}_{S} \cdot \tau\right\rangle_{\Gamma} \\
& -\left(p_{S}, \operatorname{div} \mathbf{w}_{S}\right)_{\Omega_{S}}+\left\langle p_{D}, \mathbf{w}_{S} \cdot \mathbf{n}_{S}\right\rangle_{\Gamma}=\left(\mathbf{f}, \mathbf{w}_{S}\right)_{\Omega_{S}}, \\
& \left(\operatorname{div} \mathbf{v}_{S}, q_{S}\right)_{\Omega_{S}}=0,
\end{aligned}
$$

$\forall \mathbf{w}_{S} \in \mathbf{H}_{\Gamma_{S}}^{1}\left(\Omega_{S}\right), q_{S} \in L^{2}\left(\Omega_{S}\right)$.

Similarly, taking $\delta>0$ and testing the Darcy equations (2) by $\mathbf{w}_{D} \in \mathbf{H}_{\Gamma_{D, 1}}^{1}\left(\Omega_{D}\right), q_{D} \in L^{2}\left(\Omega_{D}\right)$, respectively, together with the weighted grad-div term we obtain the weak formulation of Darcy equations: find $\mathbf{v}_{D} \in \mathbf{H}_{\Gamma_{D}}^{1}\left(\Omega_{D}\right), p_{D} \in H_{D, 2}^{1}\left(\Omega_{D}\right)$ such that

$$
\begin{aligned}
& \left(K^{-1} \mathbf{v}_{D}, \mathbf{w}_{D}\right)_{\Omega_{D}}+\left(\nabla p_{D}, \mathbf{w}_{D}\right)_{\Omega_{D}} \\
& \quad+\delta\left(\operatorname{div} \mathbf{v}_{D}, \operatorname{div} \mathbf{w}_{D}\right)_{\Omega_{D}}=\delta\left(g, \operatorname{div} \mathbf{w}_{D}\right)_{\Omega_{D}}, \\
& -\left(\mathbf{v}_{D}, \nabla q_{D}\right)_{\Omega_{D}}+\left\langle\mathbf{v}_{D} \cdot \mathbf{n}_{D}, q_{D}\right\rangle_{\Gamma}=\left(g, q_{D}\right)_{\Omega_{D}} .
\end{aligned}
$$

Summing up (11) and (12) the weak form of the coupled problem is given by the following: find $\mathbf{v}_{S} \in \mathbf{H}_{\Gamma_{S}}^{1}\left(\Omega_{S}\right), p_{S} \in$ $L^{2}\left(\Omega_{S}\right), \mathbf{v}_{D} \in \mathbf{H}_{\Gamma_{D}}^{1}\left(\Omega_{D}\right)$, and $p_{D} \in L^{2}\left(\Omega_{D}\right)$ such that

$$
\begin{aligned}
& \left(2 v D\left(\mathbf{v}_{S}\right), D\left(\mathbf{w}_{S}\right)\right)_{\Omega_{S}}-\left(p_{S}, \operatorname{div} \mathbf{w}_{S}\right)_{\Omega_{S}} \\
& +\left(K^{-1} \mathbf{v}_{D}, \mathbf{w}_{D}\right)_{\Omega_{D}}+\left(\nabla p_{D}, \mathbf{w}_{D}\right)_{\Omega_{D}} \\
& +\delta\left(\operatorname{div} \mathbf{v}_{D}, \operatorname{div} \mathbf{w}_{D}\right)_{\Omega_{D}}+\frac{\nu \alpha}{\sqrt{\tilde{k}}}\left\langle\mathbf{v}_{S} \cdot \tau, \mathbf{w}_{S} \cdot \tau\right\rangle_{\Gamma} \\
& \quad+\left\langle p_{D}, \mathbf{w}_{S} \cdot \mathbf{n}_{S}\right\rangle_{\Gamma}=\left(\mathbf{f}, \mathbf{w}_{S}\right)_{\Omega_{S}}+\delta\left(g, \operatorname{div} \mathbf{w}_{D}\right)_{\Omega_{D}}, \\
& \left(\operatorname{div} \mathbf{v}_{S}, q_{S}\right)_{\Omega_{S}}-\left(\mathbf{v}_{D}, \nabla q_{D}\right)_{\Omega_{D}} \\
& \quad-\left\langle\mathbf{v}_{S} \cdot \mathbf{n}_{S}, q_{D}\right\rangle_{\Gamma}=\left(g, q_{D}\right)_{\Omega_{D}} .
\end{aligned}
$$


To analyze the weak formulation of the coupled problem we introduce the following spaces

$$
\begin{aligned}
\mathbf{V} & =\left\{\mathbf{v} \in \mathbf{H}(\operatorname{div}, \Omega) \mid \mathbf{v}_{S} \in\left(H^{1}\left(\Omega_{S}\right)\right)^{d}, \mathbf{v}_{S}\right. \\
& \left.=0 \text { on } \Gamma_{S}, \mathbf{v} \cdot \mathbf{n}_{D}=0 \text { on } \Gamma_{D, 1}\right\}, \\
Q & =\left\{q \in L^{2}(\Omega) \mid p_{D} \in H^{1}\left(\Omega_{D}\right), p=0 \in \Gamma_{D, 2}\right\}, \\
X & =\mathbf{V} \times Q .
\end{aligned}
$$

The velocity and pressure spaces $\mathbf{V}$ and $Q$ are equipped with the natural norms

$$
\begin{aligned}
& \|\mathbf{v}\|_{\mathbf{V}}=\left(\|\nabla \mathbf{v}\|_{\Omega_{S}}^{2}+\|\mathbf{v}\|_{\Omega_{D}}^{2}+\|\operatorname{div} \mathbf{v}\|_{\Omega_{D}}^{2}\right)^{1 / 2}, \\
& \|p\|_{Q}=\left(\|p\|_{\Omega_{S}}^{2}+\|\nabla p\|_{\Omega_{D}}^{2}\right)^{1 / 2} .
\end{aligned}
$$

Further, due to the positive definiteness of $K$ with respect to the $L^{2}\left(\Omega_{D}\right)$ norm $\|\cdot\|_{\Omega_{D}}$, there exist positive real numbers $k_{1}$ and $k_{2}$ such that

$$
k_{1}\|\mathbf{v}\|_{\Omega_{D}}^{2} \leq\left(K^{-1} \mathbf{v}, \mathbf{v}\right)_{\Omega_{D}} \leq k_{2}\|\mathbf{v}\|_{\Omega_{D}}^{2}, \quad \forall \mathbf{v} \in \mathbf{V}
$$

Next, we define the bilinear forms for $\mathbf{v}=\left(\mathbf{v}_{S}, \mathbf{v}_{D}\right), \mathbf{w}=$ $\left(\mathbf{w}_{S}, \mathbf{w}_{D}\right)$ in $\mathbf{V}$ and $p=\left(p_{S}, p_{D}\right), q=\left(q_{S}, q_{D}\right)$ in $Q$ on the two parts of the domain by

$$
\begin{aligned}
\mathscr{A}_{S}(\mathbf{v}, p ; \mathbf{w}, q)= & \left(2 \nu D\left(\mathbf{v}_{S}\right), D\left(\mathbf{w}_{S}\right)\right)_{\Omega_{S}} \\
& +\frac{v \alpha}{\sqrt{\widetilde{k}}}\left\langle\mathbf{v}_{S} \cdot \tau, \mathbf{w}_{S} \cdot \tau\right\rangle_{\Gamma} \\
& -\left(p_{S}, \operatorname{div} \mathbf{w}_{S}\right)_{\Omega_{S}} \\
& +\left(\operatorname{div} \mathbf{v}_{S}, q_{S}\right)_{\Omega_{S}}, \\
\mathscr{A}_{D}(\mathbf{v}, p ; \mathbf{w}, q)= & \left(K^{-1} \mathbf{v}_{D}, \mathbf{w}_{D}\right)_{\Omega_{D}} \\
& +\delta\left(\operatorname{div} \mathbf{v}_{D}, \operatorname{div} \mathbf{w}_{D}\right)_{\Omega_{D}} \\
& +\left(\nabla p_{D}, \mathbf{w}_{D}\right)_{\Omega_{D}}-\left(\mathbf{v}_{D}, \nabla q_{D}\right)_{\Omega_{D}} .
\end{aligned}
$$

Hence, the bilinear form for the coupled problem is the sum of $\mathscr{A}_{S}(\mathbf{v}, p ; \mathbf{w}, q), \mathscr{A}_{D}(\mathbf{v}, p ; \mathbf{w}, q)$, and terms to enforce the continuity of the normal part of the velocities across the interface.

$$
\begin{aligned}
\mathscr{A}(\mathbf{v}, p ; \mathbf{w}, q)= & \mathscr{A}_{S}(\mathbf{v}, p ; \mathbf{w}, q)+\mathscr{A}_{D}(\mathbf{v}, p ; \mathbf{w}, q) \\
& +\left\langle p_{D}, \mathbf{w}_{S} \cdot \mathbf{n}_{S}\right\rangle_{\Gamma}-\left\langle q_{D}, \mathbf{v}_{S} \cdot \mathbf{n}_{S}\right\rangle_{\Gamma} .
\end{aligned}
$$

Assuming, for simplicity, that $\mathbf{f}$ and $g$ are extended by zero to the whole domain, the variational formulation of the coupled Stokes-Darcy system in compact form reads as follows: find $(\mathbf{v}, p) \in \mathbf{V} \times Q$ solution of

$$
\mathscr{A}(\mathbf{v}, p ; \mathbf{w}, q)=\mathscr{F}(\mathbf{w}, q), \quad \forall(\mathbf{w}, q) \in \mathbf{V} \times Q,
$$

with

$$
\mathscr{F}(\mathbf{w}, q)=\left(\mathbf{f}, \mathbf{w}_{S}\right)_{\Omega}+\left(g, q_{D}\right)_{\Omega}+\delta\left(g, \operatorname{div} \mathbf{w}_{D}\right)_{\Omega} .
$$

It can easily be shown that a sufficiently regular solution $(\mathbf{v}, p) \in \mathbf{V} \times \mathbf{Q}$ of (19) such that $\mathbf{v}_{S} \in H^{2}\left(\Omega_{S}\right)^{d}, \mathbf{v}_{D} \in$ $H^{1}\left(\Omega_{D}\right)^{d}, p \in H^{1}\left(\Omega_{S} \cup \Omega_{D}\right)$ is also a classical solution of (1) and (2). We note that there is an alternative variational formulation to the one given here called $H$ (div)-formulation. The latter uses the term $-(p, \operatorname{div} \mathbf{w})_{\Omega_{D}}+(\operatorname{div} \mathbf{v}, q)_{\Omega_{D}}$ instead of $(\mathbf{w}, \nabla p)_{\Omega_{D}}-(\mathbf{v}, \nabla q)_{\Omega_{D}}[4]$.

The existence and uniqueness of the solution of problem (19) follows from Brezzi's conditions for saddle point problems [11]; namely,

$$
\begin{aligned}
A(\mathbf{v}, p ; \mathbf{v}, p) \geq \tilde{\gamma}\|\mathbf{v}\|_{\mathbf{V}}^{2}, & \\
& \forall v \in \mathbf{V}, \tilde{\gamma}>0,
\end{aligned}
$$

$$
\begin{gathered}
\inf _{q \in L^{2}\left(\Omega_{S}\right)} \sup _{\mathbf{v} \in H^{1}\left(\Omega_{S}\right)^{d}} \frac{(\operatorname{div} \mathbf{v}, q)_{\Omega_{S}}}{\|\nabla \mathbf{v}\|_{\Omega_{S}}\|q\|_{\Omega_{S}}} \geq \beta_{S}, \\
\inf _{q \in H^{1}\left(\Omega_{D}\right)} \sup _{\mathbf{v} \in L^{2}\left(\Omega_{D}\right)^{d}} \frac{-(\mathbf{v}, \nabla q)_{\Omega_{D}}}{\|\mathbf{v}\|_{\Omega_{D}}\|\nabla q\|_{\Omega_{D}}} \geq \beta_{D} .
\end{gathered}
$$

with positive constants $\beta_{S}$ and $\beta_{D}$ [7].

The following lemma is needed in the analysis below and is a consequence of the continuous inf-sup conditions (23) [10].

Lemma 1. For every $(\mathbf{v}, p) \in X$ there is $\mathbf{w} \in \mathbf{V}$ such that $\mathbf{w}_{S}$. $\mathbf{n}_{S}=0$ on $\Gamma$, satisfying

$$
\begin{gathered}
\mathscr{A}(\mathbf{v}, p ; \mathbf{w}, 0) \geq c_{2}\|p\|_{\mathrm{Q}}^{2}-c_{1}\|\mathbf{v}\|_{\mathbf{V}}^{2}, \\
\|\mathbf{w}\|_{\mathbf{v}} \leq c_{3}\|p\|_{Q},
\end{gathered}
$$

with positive constants $c_{1}, c_{2}$, and $c_{3}$.

Proof. Let $(\mathbf{v}, p) \in X$. Then, due to Stokes inf-sup condition there exists $\mathbf{w}_{S} \in H^{1}\left(\Omega_{S}\right)^{d}$ with $\mathbf{w}_{S}=\mathbf{0}$ on $\Gamma_{S}$ and $\mathbf{w}_{S} \cdot \mathbf{n}=0$ on $\Gamma$ such that

$$
\begin{aligned}
&-\left(\operatorname{div} \mathbf{w}_{S}, p\right)_{\Omega_{S}}=\|p\|_{\Omega_{S}}^{2}, \\
&\left\|\nabla \mathbf{w}_{S}\right\|_{\Omega_{S}} \leq c_{S}\|p\|_{\Omega_{S}} .
\end{aligned}
$$

For the Darcy equation, due to the condition $p=0$ on $\Gamma_{D, 2}$, there exists $\mathbf{w}_{D} \in H^{1}\left(\Omega_{D}\right)^{d}$ with $\mathbf{w}_{D} \cdot \mathbf{n}=0$ on $\Gamma_{D, 2}$ and $\Gamma$, such that

$$
\begin{aligned}
-\left(\operatorname{div} \mathbf{w}_{D}, p\right)_{\Omega_{D}} & =\|\nabla p\|_{\Omega_{D}}^{2}, \\
\left\|\nabla \mathbf{w}_{D}\right\|_{\Omega_{D}} & \leq c_{D}\|\nabla p\|_{\Omega_{D}} .
\end{aligned}
$$

Define

$$
\mathbf{w}= \begin{cases}\mathbf{w}_{S} & \text { in } \Omega_{S} \\ \mathbf{w}_{D} & \text { in } \Omega_{D},\end{cases}
$$


and then

$$
\begin{aligned}
\mathscr{A}(\mathbf{v}, p ; \mathbf{w}, 0)= & (2 v D(\mathbf{v}), D(\mathbf{w}))_{\Omega_{S}}-(p, \operatorname{div} \mathbf{w})_{\Omega_{S}} \\
& +\left(K^{-1} \mathbf{v}, \mathbf{w}\right)_{\Omega_{D}}+(\nabla p, \mathbf{w})_{\Omega_{D}} \\
& +\delta(\operatorname{div} \mathbf{v}, \operatorname{div} \mathbf{w})_{\Omega_{D}} \\
\geq & -2 v\|D(\mathbf{v})\|_{\Omega_{S}}\|D(\mathbf{w})\|_{\Omega_{S}}+\|p\|_{\Omega_{S}}^{2} \\
& -k_{2}\|\mathbf{v}\|_{\Omega_{D}}\left\|\mathbf{w}_{D}\right\|_{\Omega_{D}}+\|\nabla p\|_{\Omega_{D}}^{2} \\
& -\delta\|\operatorname{div} \mathbf{v}\|_{\Omega_{D}}\|\operatorname{div} \mathbf{w}\|_{\Omega_{D}} \\
\geq & -2 v\|\nabla \mathbf{v}\|_{\Omega_{S}}\|\nabla \mathbf{w}\|_{\Omega_{S}}+\|p\|_{\Omega_{S}}^{2} \\
& -k_{2}\|\mathbf{v}\|_{\Omega_{D}}\left\|\mathbf{w}_{D}\right\|_{\Omega_{D}}+\|\nabla p\|_{\Omega_{D}}^{2} \\
& -\delta\|\operatorname{div} \mathbf{v}\|_{\Omega_{D}}\|\nabla \mathbf{w}\|_{\Omega_{D}} \\
\geq & -2 v c_{S}\|\nabla \mathbf{v}\|_{\Omega_{S}}\|p\|_{\Omega_{S}}+\|p\|_{\Omega_{S}}^{2} \\
& -c_{p} c_{D} k_{2}\|\mathbf{v}\|_{\Omega_{D}}\|\nabla p\|_{\Omega_{D}}+\|\nabla p\|_{\Omega_{D}}^{2} \\
& -\delta c_{D}\|\operatorname{div} \mathbf{v}\|_{\Omega_{D}}\|\nabla p\|_{\Omega_{D}},
\end{aligned}
$$

where $c_{p}$ denote the Poincaré constant.

Then, using Young's inequality we obtain

$$
\begin{aligned}
\mathscr{A}(\mathbf{v}, p ; \mathbf{w}, 0) \geq & -\frac{v c_{S}}{\varepsilon_{1}}\|\nabla \mathbf{v}\|_{\Omega_{S}}^{2}+\left(1-\nu c_{S} \varepsilon_{1}\right)\|p\|_{\Omega_{S}}^{2} \\
& -\frac{c_{p} c_{D} k_{2}}{2 \varepsilon_{2}}\|\mathbf{v}\|_{\Omega_{D}}^{2} \\
& +\left(1-\frac{c_{p} c_{D} k_{2} \varepsilon_{2}}{2}-\frac{\delta c_{D} \varepsilon_{3}}{2}\right)\|\nabla p\|_{\Omega_{D}}^{2} \\
& -\frac{\delta c_{D}}{2 \varepsilon_{3}}\|\operatorname{div} \mathbf{v}\|_{\Omega_{D}}^{2} .
\end{aligned}
$$

Choosing $\varepsilon_{1}, \varepsilon_{2}, \varepsilon_{3}$ positive constants such that

$$
\begin{aligned}
& \varepsilon_{1}<\frac{1}{v c_{S}}, \\
& \varepsilon_{2}<\frac{2}{c_{p} c_{D} k_{2}}, \\
& \varepsilon_{3}<\frac{2-c_{p} c_{D} k_{2} \varepsilon_{2}}{\delta c_{D}},
\end{aligned}
$$

we obtain the required result

$$
\mathscr{A}(\mathbf{v}, p ; \mathbf{w}, 0) \geq c_{2}\|p\|_{\mathrm{Q}}^{2}-c_{1}\|\mathbf{v}\|_{\mathbf{v}}^{2},
$$

where

$$
\begin{aligned}
& c_{1}=\max \left\{\frac{\nu c_{S}}{\varepsilon_{1}}, \frac{c_{p} c_{D} k_{2}}{2 \varepsilon_{2}}, \frac{\delta c_{D}}{2 \varepsilon_{3}}\right\}, \\
& c_{2}=\min \left\{1-\nu c_{S} \varepsilon_{1}, 1-\frac{c_{p} c_{D} k_{2} \varepsilon_{2}}{2}-\frac{\delta c_{D} \varepsilon_{3}}{2}\right\} .
\end{aligned}
$$

In addition, we also have

$$
\begin{aligned}
\|\mathbf{w}\|_{\mathbf{V}}^{2} & =\|\nabla \mathbf{w}\|_{\Omega_{S}}^{2}+\|\mathbf{w}\|_{\Omega_{D}}^{2}+\|\operatorname{div} \mathbf{w}\|_{\Omega_{D}}^{2} \\
& \leq c_{S}^{2}\|p\|_{\Omega_{S}}^{2}+c_{D}^{2}\left(c_{p}^{2}+1\right)\|\nabla p\|_{\Omega_{D}}^{2} \leq c_{3}\|p\|_{Q},
\end{aligned}
$$

where $c_{3}^{2}=\max \left\{c_{S}^{2}, c_{D}^{2}\left(c_{p}^{2}+1\right)\right\}$.

\section{Finite Element Discretization}

Let $\mathscr{T}_{h}$ be a shape-regular partition of quadrilaterals for $d=$ 2 or hexahedra for $d=3[12,13]$. The diameter of element $T \in \mathscr{T}_{h}$ will be denoted by $h_{T}$ and the global mesh size is defined by $h:=\max \left\{h_{T}: T\right.$ in $\left.\mathscr{T}_{h}\right\}$. Let $\widehat{T}:=(-1 ; 1)^{d}$ be the reference element, $F_{T}$ the mapping from $\widehat{T}$ to element $T$, and $Q^{r}(\widehat{T})$ the space of all polynomials on $\widehat{T}$ with maximal degree $r \geq 0$ in each coordinate. We assume that the mesh $\mathscr{T}_{h}$ is obtained from a coarser mesh $\mathscr{T}_{2 h}$ by global refinement. Hence, $\mathscr{T}_{2 h}$ consists of patches of elements of $\mathscr{T}_{h}$. We define the finite element space

$$
\begin{aligned}
X_{h}^{r} & :=\left\{v \in C\left(\Omega_{S}\right) \cup C\left(\Omega_{D}\right):\left.v\right|_{T}\right. \\
& \left.\circ F_{T} \text { in } Q_{r}(\widehat{T}), \forall T \in \mathscr{T}_{h}\right\} .
\end{aligned}
$$

For the discrete spaces $\mathbf{V}_{h}$ and $Q_{h}$ we use the equal-order finite element functions that are continuous in $\Omega_{S}$ and $\Omega_{D}$ and piecewise polynomials of degree $r \geq 1$.

$$
\begin{aligned}
& \mathbf{V}_{h}=\left(X_{h}^{r}\right)^{d} \cap \mathbf{V}, \\
& Q_{h}=X_{h}^{r} \cap Q \cap H^{1}(\Omega) .
\end{aligned}
$$

We define the Scott-Zhang interpolation operator which preserves the boundary condition [13], as $j_{r}^{h}: H^{1}(\Omega) \rightarrow X_{h}^{r}$ with stability and interpolation properties, respectively, as

$$
\begin{aligned}
& \left\|\nabla j_{r}^{h} \phi\right\|_{\Omega} \leq c_{s}|\phi|_{1, \Omega}, \quad \phi \in H^{1}(\Omega) . \\
& \left\|\phi-j_{r}^{h} \phi\right\|_{m, \Omega} \leq c_{i} h^{r+1-m}|\phi|_{r+1, \Omega}, \\
& \quad \phi \in H^{r+1}(\Omega), m=0 \text { or } 1,
\end{aligned}
$$

where $c_{i}, c_{s}$ are positive constants.

We will also use the inverse inequality

$$
\left(\sum_{T \in \mathscr{T}_{h}} h_{T}^{2}\|\nabla \phi\|_{T}\right)^{1 / 2} \leq c_{I}\|\phi\|_{\Omega}, \quad \forall \phi \in H^{1}(\Omega) .
$$

Similarly, for vector functions we define the interpolation operator

$$
\mathbf{j}_{r}^{h}: H^{1}(\Omega)^{d} \longrightarrow\left(X_{h}^{r}\right)^{d},
$$

with interpolation and stability properties as above.

It is known that the standard Galerkin discretizations of the Darcy system are not stable for equal-order elements. This instability stems from the violation of the discrete analogue 
on to the inf-sup condition. One possibility to circumvent this condition is to work with a modified bilinear form $\mathscr{A}_{h}(\cdot ; \cdot)$ by adding a stabilization term $\mathcal{S}_{h}(\cdot ; \cdot)$; that is,

$$
\mathscr{A}_{h}\left(\mathbf{v}_{h}, p_{h} ; \mathbf{w}, q\right)=\mathscr{A}\left(\mathbf{v}_{h}, p_{h} ; \mathbf{w}, q\right)+\mathcal{S}_{h}\left(p_{h} ; q\right),
$$

such that the stabilized discrete problem reads

$$
\mathscr{A}_{h}\left(\mathbf{v}_{h}, p_{h} ; \mathbf{w}, q\right)=\mathscr{F}(\mathbf{w}, q) \quad \forall(\mathbf{w}, q) \in \mathbf{V}_{h} \times Q_{h} .
$$

Unlike in [10] where a combination of a generalized mini element and local projection (LPS) is analyzed and in [14] where a method based on two local Gauss integrals for the Stokes equations is used, here we will analyze the problem using a subgrid method $[12,15,16]$.

For this method the filter, with respect to the global Lagrange interpolant $I_{2 h}$, onto a coarser mesh $\mathscr{T}_{2 h}$ is used. Defining $\kappa_{2 h}=I-I_{2 h}$ the subgrid stabilization term reads

$$
\mathcal{S}_{h}\left(p_{h} ; q\right)=\sum_{M \in \mathscr{T}_{2 h}} h_{M}\left(\gamma \nabla \kappa_{2 h} p_{h}, \nabla \kappa_{2 h} q\right)_{M}, \quad r \geq 1,
$$

where $\gamma$ is patchwise constant.

A more attractive method from the computational point is obtained using only the fine mesh with smaller stencil. Defining $\kappa_{h}=I-I_{h}$ the subgrid stabilization term reads

$$
\mathcal{S}_{h}\left(p_{h} ; q\right)=\sum_{K \in \mathscr{T}_{h}} h_{K}\left(\gamma \nabla \kappa_{h} p_{h}, \nabla \kappa_{h} q\right)_{K}, \quad r \geq 2 .
$$

Next, we prove the stability of the discrete coupled StokesDarcy problem with respect to the norm

$$
\|(\mathbf{v}, p)\|_{h}=\left(\|\mathbf{v}\|_{\mathbf{V}}^{2}+\|p\|_{Q}^{2}+\mathcal{S}_{h}(p ; p)\right)^{1 / 2} .
$$

\section{Stability}

Theorem 2. Let $\mathscr{T}_{h}$ be a quasi-regular partition [13]. Then, the following discrete inf-sup condition holds for some positive constant $\widetilde{\beta}$ independent of the mesh size $h$.

$$
\begin{aligned}
& \inf _{\left(\mathbf{v}_{h}, p_{h}\right) \in \mathbf{V}_{h} \times Q_{h} \backslash\{0,0\}} \sup _{\left(\mathbf{w}_{h}, q_{h}\right) \in \mathbf{V}_{h} \times Q_{h} \backslash\{0,0\}} \frac{\mathscr{A}\left(\mathbf{v}_{h}, p_{h} ; \mathbf{w}_{h}, q_{h}\right)}{\left\|\left(\mathbf{v}_{h}, p_{h}\right)\right\|_{h}\left\|\left(\mathbf{w}_{h}, q_{h}\right)\right\|_{h}} \\
& \geq \widetilde{\beta} .
\end{aligned}
$$

Proof. First, let $\left(\mathbf{v}_{h}, p_{h}\right) \in \mathbf{V}_{h} \times Q_{h}$, and then the diagonal testing combined with Korn's inequality and the positivity of $K^{-1}$ give

$$
\begin{aligned}
\mathscr{A}_{h}\left(\mathbf{v}_{h}, p_{h} ; \mathbf{v}_{h}, p_{h}\right) & =\mathscr{A}\left(\mathbf{v}_{h}, p_{h} ; \mathbf{v}_{h}, p_{h}\right)+\mathcal{S}_{h}\left(p_{h} ; p_{h}\right) \\
& \geq \widetilde{\alpha}\|\mathbf{v}\|_{\mathbf{V}}^{2}+\mathcal{S}_{h}\left(p_{h} ; p_{h}\right) .
\end{aligned}
$$

In addition, let $\mathbf{w}$ be as in Lemma 1, corresponding to $\left(\mathbf{v}_{h}, p_{h}\right) \in \mathbf{V}_{h} \times Q_{h}$, and set $\mathbf{z}=\mathbf{j}_{r}^{h} \mathbf{w}-\mathbf{w}$. Then,

$$
\begin{aligned}
\mathscr{A}\left(\mathbf{v}_{h}, p_{h} ; \mathbf{j}_{r}^{h} \mathbf{w}, 0\right)= & \mathscr{A}\left(\mathbf{v}_{h}, p_{h} ; \mathbf{w}, 0\right)+\mathscr{A}\left(\mathbf{v}_{h}, p_{h} ; \mathbf{z}, 0\right) \\
\geq & c_{2}\left\|p_{h}\right\|_{Q}^{2}-c_{1}\left\|\mathbf{v}_{h}\right\|_{\mathbf{v}}^{2} \\
& +\mathscr{A}_{S}\left(\mathbf{v}_{h}, p_{h} ; \mathbf{z}, 0\right) \\
& +\mathscr{A}_{D}\left(\mathbf{v}_{h}, p_{h} ; \mathbf{z}, 0\right)
\end{aligned}
$$

Next, we estimate $\mathscr{A}_{S}\left(\mathbf{v}_{h}, p_{h} ; \mathbf{z}, 0\right)$ and $\mathscr{A}_{D}\left(\mathbf{v}_{h}, p_{h} ; \mathbf{z}, 0\right)$ as follows:

$$
\begin{aligned}
\mathscr{A}_{S}\left(\mathbf{v}_{h}, p_{h} ; \mathbf{z}, 0\right)= & \left(2 \nu D\left(\mathbf{v}_{h}\right), D(\mathbf{z})\right)_{\Omega_{S}}+\left(\nabla p_{h}, \mathbf{z}\right)_{\Omega_{S}} \\
& +\frac{\nu \alpha}{\sqrt{\tilde{k}}}\left\langle\mathbf{v}_{h S} \cdot \tau, \mathbf{z}_{S} \cdot \tau\right\rangle_{\Gamma},
\end{aligned}
$$

where the first two terms are bounded using Cauchy inequality together with the interpolation, stability, and inverse inequalities

$$
\begin{aligned}
& \left|\left(\nu D\left(\mathbf{v}_{h}\right), D(\mathbf{z})\right)_{\Omega_{S}}\right| \leq \nu\left\|D\left(\mathbf{v}_{h}\right)\right\|_{\Omega_{S}}\|D(\mathbf{z})\|_{\Omega_{S}} \\
& \leq v\left\|\mathbf{v}_{h}\right\|_{\mathbf{V}}\|\nabla \mathbf{z}\|_{\Omega_{S}} \leq v c_{i}\left\|\mathbf{v}_{h}\right\|_{\mathbf{V}}\|\nabla \mathbf{w}\|_{\Omega_{S}} \\
& \leq v c_{3} c_{i}\left\|\mathbf{v}_{h}\right\|_{\mathbf{V}}\left\|p_{h}\right\|_{Q}, \\
& \left(\nabla p_{h}, \mathbf{z}\right)_{\Omega_{S}} \leq\left(\sum_{T \in \mathscr{T}_{h}, T \subset \Omega_{S}} h_{T}^{-2}\|\mathbf{z}\|_{T}^{2}\right)^{1 / 2} \\
& \cdot\left(\sum_{T \in T_{h}, T \subset \Omega_{S}} h_{T}^{2}\left\|\nabla p_{h}\right\|_{T}^{2}\right)^{1 / 2} \\
& \quad \leq\left(\sum_{T \in T_{h}, T \subset \Omega_{S}} h_{T}^{-2} h_{T}^{2 r}\|\nabla \mathbf{w}\|_{T}^{2}\right)^{1 / 2} c_{I}\left\|p_{h}\right\|_{\Omega S} \\
& \quad \leq c c_{i} c_{I}\|\nabla \mathbf{w}\|_{\Omega_{S}}\left\|p_{h}\right\|_{\Omega_{S}} \leq c c_{i} c_{I} c_{3}\left\|p_{h}\right\|_{Q}^{2} .
\end{aligned}
$$

The boundary term is bounded using the trace theorem and the $H^{1}$ - stability by

$$
\begin{aligned}
& \left|\frac{\nu \alpha}{\sqrt{\tilde{k}}}\left\langle\mathbf{v}_{h_{S}} \cdot \tau, \mathbf{z}_{S} \cdot \tau\right\rangle_{\Gamma}\right| \leq c_{\Gamma}^{2} \frac{\nu \alpha}{\sqrt{\tilde{k}}}\left\|\mathbf{v}_{h}\right\|_{\mathbf{V}}\|\nabla \mathbf{z}\|_{\Omega_{S}} \\
& \quad \leq c_{\Gamma}^{2} c_{s} c_{3} \frac{\nu \alpha}{\sqrt{\tilde{k}}}\left\|\mathbf{v}_{h}\right\|_{\mathbf{V}}\left\|p_{h}\right\|_{Q} .
\end{aligned}
$$

Hence, by Young inequality with

$$
\begin{aligned}
\epsilon_{1} & =\frac{c_{2}}{8 \nu c_{i} c_{3}}, \\
\epsilon_{2} & =\frac{c_{2} \sqrt{\tilde{k}}}{8 \nu \alpha c_{\Gamma}^{2} c_{s} c_{3}}
\end{aligned}
$$

we obtain

$$
\mathscr{A}_{S}\left(\mathbf{v}_{h}, p_{h} ; \mathbf{z}, 0\right) \leq \frac{c_{2}}{8} c_{4}\left\|p_{h}\right\|_{Q}^{2}+c_{4}\left\|\mathbf{v}_{h}\right\|_{\mathbf{V}}^{2},
$$

where $c_{4}=\left(4\left(\nu c_{3} c_{i}\right)^{2}+0.25\left(c_{\Gamma}^{2} c_{s} c_{3}\right)^{2}\right) / c_{2}$. 
For the Darcy bilinear form we have

$$
\begin{aligned}
& \mathscr{A}_{D}\left(\mathbf{v}_{h}, p_{h} ; \mathbf{z}, 0\right)=\left(K^{-1} \mathbf{v}_{h}, \mathbf{z}\right)_{\Omega_{D}}+\delta\left(\operatorname{div} \mathbf{v}_{h}, \operatorname{div} \mathbf{z}\right)_{\Omega_{D}} \\
& +\left(\nabla p_{h}, \mathbf{z}\right)_{\Omega_{D}} \\
& =\left(K^{-1} \mathbf{v}_{h}, \mathbf{z}\right)_{\Omega_{D}}+\delta\left(\operatorname{div} \mathbf{v}_{h}, \operatorname{div} \mathbf{z}\right)_{\Omega_{D}} \\
& +\left(\nabla\left(p_{h}-\kappa_{2 h} p_{h}\right), \mathbf{z}\right)_{\Omega_{D}} \\
& +\left(\nabla \kappa_{2 h} p_{h}, \mathbf{z}\right)_{\Omega_{D}} \\
& \leq\left\|K^{-1} \mathbf{v}_{h}\right\|_{\Omega_{D}}\|\mathbf{z}\|_{\Omega_{D}} \\
& +\delta\left\|\operatorname{div} \mathbf{v}_{h}\right\|_{\Omega_{D}}\|\mathbf{z}\|_{\Omega_{D}} \\
& +\left\|\nabla\left(p_{h}-\kappa_{2 h} p_{h}\right)\right\|_{\Omega_{D}}\|\mathbf{z}\|_{\Omega_{D}} \\
& +\left\|\nabla \kappa_{2 h} p_{h}\right\|_{\Omega_{D}}\|\mathbf{z}\|_{\Omega_{D}} \\
& \leq k_{2}\left\|\mathbf{v}_{h}\right\|_{\Omega_{D}} c_{i}\|\mathbf{w}\|_{\Omega_{D}} \\
& +\delta\left\|\operatorname{div} \mathbf{v}_{h}\right\|_{\Omega_{D}}\left(1+c_{s}\right)\|\mathbf{w}\|_{\Omega_{D}} \\
& +\left\|\nabla\left(p_{h}-\kappa_{2 h} p_{h}\right)\right\|_{\Omega_{D}} c_{i}\|\mathbf{w}\|_{\Omega_{D}} \\
& +c_{s}\left\|\nabla p_{h}\right\|_{\Omega_{D}} \\
& \leq k_{2} c_{i} c_{3}\left\|p_{h}\right\|_{Q} \\
& +\delta c_{3}\left(1+c_{s}\right)\left\|\operatorname{div} \mathbf{v}_{h}\right\|_{\Omega_{D}}\left\|p_{h}\right\|_{Q} \\
& +c_{i} c_{3}\left\|\nabla\left(p_{h}-\kappa_{2 h} p_{h}\right)\right\|_{\Omega_{D}} \\
& +c_{s}\left\|p_{h}\right\|_{Q} \text {. }
\end{aligned}
$$

Then, by Young inequality and (52) we obtain

$$
\begin{aligned}
\mathscr{A}_{h}\left(\mathbf{v}_{h}, p_{h} ; \mathbf{j}_{r}^{h} \mathbf{w}, 0\right) \geq & \frac{5 c_{2}}{8}\left\|p_{h}\right\|_{\mathrm{Q}}^{2} \\
& -C\left(\left\|\mathbf{v}_{h}\right\|_{\mathbf{V}}^{2}+\mathcal{S}_{h}\left(p_{h} ; p_{h}\right)\right) .
\end{aligned}
$$

Scaling $\mathbf{j}_{r}^{h} \mathbf{w}$ we obtain

$$
\begin{aligned}
\mathscr{A}_{h}\left(\mathbf{v}_{h}, p_{h} ; \mathbf{j}_{r}^{h} \mathbf{w}, 0\right) \geq & \left\|p_{h}\right\|_{\mathrm{Q}}^{2} \\
& -C_{1}\left(\left\|\mathbf{v}_{h}\right\|_{\mathbf{v}}^{2}+\mathcal{S}_{h}\left(p_{h} ; p_{h}\right)\right) .
\end{aligned}
$$

Choosing $\left(\mathbf{w}_{h}, q_{h}\right)=\left(\mathbf{v}_{h}, p_{h}\right)+\left(1 /\left(1+C_{1}\right)\right)\left(\mathbf{j}_{r}^{h} \mathbf{w}, 0\right)$ we obtain

$$
\begin{aligned}
\mathscr{A}_{h}\left(\mathbf{v}_{h}, p_{h} ; \mathbf{w}_{h}, q_{h}\right) \geq & \left\|\mathbf{v}_{h}\right\|_{\mathbf{V}}^{2}+\frac{1}{1+C_{1}}\left\|p_{h}\right\|_{Q}^{2} \\
& -\frac{C_{1}}{1+C_{1}}\left\|\mathbf{v}_{h}\right\|_{\mathbf{V}}^{2} \\
= & \frac{1}{1+C_{1}}\left(\left\|\mathbf{v}_{h}\right\|_{\mathbf{V}}^{2}+\left\|p_{h}\right\|_{Q}^{2}\right) \\
= & \frac{1}{1+C_{1}}\left\|\left(\mathbf{v}_{h}, p_{h}\right)\right\|_{h}^{2},
\end{aligned}
$$

$\left\|\mathbf{w}_{h}, q_{h}\right\|_{h} \leq\left\|\left(\mathbf{v}_{h}, p_{h}\right)\right\|_{h}$

$$
\begin{gathered}
+\frac{1}{1+C_{1}}\left\|\left(\mathbf{j}_{r}^{h} \mathbf{w}, 0\right)\right\|_{h} \\
\leq\left\|\left(\mathbf{v}_{h}, p_{h}\right)\right\|_{h}+C_{2}\left\|\nabla \mathbf{j}_{r}^{h} \mathbf{w}\right\|_{\Omega} \\
\leq C_{3}\left\|\left(\mathbf{v}_{h}, p_{h}\right)\right\|_{h}
\end{gathered}
$$

which implies the required result

$$
\inf _{\left(\mathbf{v}_{h}, p_{h}\right) \in \mathbf{V}_{h} \times Q_{h} \backslash\{0\}} \sup _{\left(\mathbf{w}_{h}, q_{h}\right) \in \mathbf{V}_{h} \times Q_{h} \backslash\{0\}} \frac{\mathscr{A}_{h}\left(\mathbf{v}_{h}, p_{h} ; \mathbf{w}_{h}, q_{h}\right)}{\left\|\left(\mathbf{v}_{h}, p_{h}\right)\right\|_{h}\left\|\left(\mathbf{w}_{h}, q_{h}\right)\right\|_{h}} \geq \tilde{\beta},
$$

with $\widetilde{\beta}=C_{3}^{-1} /\left(1+C_{1}\right)$.

\section{Error Analysis}

Theorem 3. Assume that the solution $(\mathbf{v}, p)$ of the StokesDarcy problem (19) is such that $\left(\mathbf{v}_{S}, p_{S}\right) \in \mathbf{V}_{S} \cap H^{r+1}\left(\Omega_{S}\right)^{d} \times$ $Q \cap H^{l+1}\left(\Omega_{S}\right),\left(\mathbf{v}_{D}, p_{D}\right) \in \mathbf{V}_{D} \cap H^{r+1}\left(\Omega_{D}\right)^{d} \times Q \cap H^{l+1}\left(\Omega_{D}\right)$, and $\left(\mathbf{v}_{h}, p_{h}\right)$ is the solution of the stabilized problem (41). Then, the following error estimate holds with constants $c_{1}, c_{2}, \ldots, c_{7}$ independent of $h$ :

$$
\begin{aligned}
\| & \left\|\left(\mathbf{v}-\mathbf{v}_{h}, p-p_{h}\right)\right\|_{h} \leq\left\{\left(c_{1} v+c_{2}\right)^{2} h^{2 r}\|\mathbf{v}\|_{r+1, \Omega_{S}}^{2}\right. \\
& +\left(c_{3} h+c_{4} \delta\right)^{2} h^{2 r}\|\mathbf{v}\|_{r+1, \Omega_{D}}^{2} \\
& +\left(c_{5}+c_{6} \gamma^{1 / 2} h^{1 / 2}+c_{7} h\right)^{2} h^{2 l}\|p\|_{l+1, \Omega_{S}}^{2} \\
& \left.+\left(c_{5}+c_{6} \gamma^{1 / 2} h^{1 / 2}+c_{7} h\right)^{2} h^{2 l}\|p\|_{l+1, \Omega_{D}}^{2}\right\}^{1 / 2} .
\end{aligned}
$$

Proof. Using the stability estimate of Theorem 3, there exists $\left(\mathbf{w}_{h}, q_{h}\right) \in \mathbf{V}_{h} \times Q_{h}$, with $\left\|\left(\mathbf{w}_{h}, q_{h}\right)\right\|_{h} \leq \widetilde{C}$ satisfying

$$
\begin{aligned}
\left\|\left(\mathbf{j}_{r}^{h} \mathbf{v}-\mathbf{v}_{h}, j_{l}^{h} p-p_{h}\right)\right\|_{h} \\
\leq \frac{1}{\widetilde{\beta}} \frac{\mathscr{A}_{h}\left(\mathbf{j}_{r}^{h} \mathbf{v}-\mathbf{v}_{h}, j_{l}^{h} p-p ; \mathbf{w}_{h}, q_{h}\right)}{\left\|\left(\mathbf{w}_{h}, q_{h}\right)\right\|_{h}} \\
\leq \frac{1}{\widetilde{\beta}} \frac{\mathscr{A}_{h}\left(\mathbf{v}-\mathbf{v}_{h}, p-p_{h} ; \mathbf{w}_{h}, q_{h}\right)}{\left\|\left(\mathbf{w}_{h}, q_{h}\right)\right\|_{h}} \\
\quad+\frac{1}{\widetilde{\beta}} \frac{\mathscr{A}_{h}\left(\mathbf{j}_{r}^{h} \mathbf{v}-\mathbf{v}, j_{l}^{h} p-p ; \mathbf{w}_{h}, q_{h}\right)}{\left\|\left(\mathbf{w}_{h}, q_{h}\right)\right\|_{h}} .
\end{aligned}
$$

Then, by Galerkin orthogonality property, the first term of (59) is bounded by

$$
\begin{gathered}
\frac{\mathscr{A}_{h}\left(\mathbf{v}-\mathbf{v}_{h}, p-p_{h} ; \mathbf{w}_{h}, q_{h}\right)}{\left\|\left(\mathbf{w}_{h}, q_{h}\right)\right\|_{h}}=\frac{\mathcal{S}_{h}\left(p ; q_{h}\right)}{\left\|\left(\mathbf{w}_{h}, q_{h}\right)\right\|_{h}} \\
\leq \frac{\mathcal{S}_{h}(p ; p)^{1 / 2} \mathcal{S}_{h}\left(q_{h} ; q_{h}\right)^{1 / 2}}{\left\|\left(\mathbf{w}_{h}, q_{h}\right)\right\|_{h}} \leq \mathcal{S}_{h}(p ; p)^{1 / 2} .
\end{gathered}
$$


Hence, the approximation properties of $\kappa_{2 h}$ and $\kappa_{h}$ imply

$$
\begin{gathered}
\frac{1}{\tilde{\beta}} \frac{\mathscr{A}_{h}\left(\mathbf{v}-\mathbf{v}_{h}, p-p_{h} ; \mathbf{w}_{h}, q_{h}\right)}{\left\|\left(\mathbf{w}_{h}, q_{h}\right)\right\|_{h}} \\
\leq \frac{1}{\widetilde{\beta}}\left\|\gamma \nabla \kappa_{2 h} p\right\|_{\Omega}\left\|\nabla \kappa_{2 h} p\right\|_{\Omega} \\
\quad \leq c_{1} \widetilde{\beta}^{-1} \gamma^{1 / 2} h^{l+1 / 2}\|p\|_{l+1, \Omega} .
\end{gathered}
$$

To estimate the second term of (59) we consider separately each individual term of the bilinear form $(1 / \widetilde{\beta}) \mathscr{A}_{h}\left(\mathbf{j}_{r}^{h} \mathbf{v}-\right.$ $\left.\mathbf{v}, j_{l}^{h} p-p ; \mathbf{w}_{h}, q_{h}\right)$.

Next, Cauchy schwarz and Poincaré inequality for the boundary terms imply

$$
\begin{aligned}
& \frac{1}{\widetilde{\beta}} \mathscr{A}_{S}\left(\mathbf{j}_{r}^{h} \mathbf{v}-\mathbf{v}, j_{l}^{h} p-p ; \mathbf{w}_{h}, q_{h}\right) \\
& \leq \widetilde{\beta}^{-1}\left[\nu\left\|\nabla\left(\mathbf{j}_{r}^{h} \mathbf{v}-\mathbf{v}\right)\right\|_{\Omega_{S}}\left\|\nabla \mathbf{w}_{h}\right\|_{\Omega_{S}}\right. \\
& +\left\|j_{l}^{h} p-p\right\|_{\Omega_{S}}\left\|\nabla \mathbf{w}_{h}\right\|_{\Omega_{S}}\left\|\nabla\left(\mathbf{j}_{r}^{h} \mathbf{v}-\mathbf{v}\right)\right\|_{\Omega_{S}}\left\|q_{h}\right\|_{\Omega_{S}} \\
& \left.+\frac{v \alpha c_{\Gamma}^{2}}{\sqrt{\tilde{k}}}\left\|\nabla\left(\mathbf{j}_{r}^{h} \mathbf{v}-\mathbf{v}\right)\right\|_{\Omega_{S}}\left\|\nabla \mathbf{w}_{h}\right\|_{\Omega_{S}}\right] \\
& \leq \widetilde{\beta}^{-1} c_{i} \widetilde{C}\left[v h^{r}\|\mathbf{v}\|_{r+1, \Omega_{S}}+h^{l+1}\|p\|_{l, \Omega_{S}}\right. \\
& \left.+h^{r}\|\mathbf{v}\|_{r+1, \Omega_{S}}+\frac{v \alpha c_{\Gamma}^{2}}{\sqrt{\widetilde{k}}} h^{r}\|\mathbf{v}\|_{r+1, \Omega_{S}}\right], \\
& \frac{1}{\widetilde{\beta}} \mathscr{A}_{D}\left(\mathbf{j}_{r}^{h} \mathbf{v}-\mathbf{v}, j_{l}^{h} p-p ; \mathbf{w}_{h}, q_{h}\right) \\
& \leq \tilde{\beta}^{-1}\left[k_{2}\left\|\mathbf{j}_{r}^{h} \mathbf{v}-\mathbf{v}\right\|_{\Omega_{D}}\left\|\mathbf{w}_{h}\right\|_{\Omega_{D}}\right. \\
& +\delta\left\|\nabla\left(\mathbf{j}_{r}^{h} \mathbf{v}-\mathbf{v}\right)\right\|_{\Omega_{D}}\left\|\operatorname{div} \mathbf{w}_{h}\right\|_{\Omega_{D}} \\
& +\left\|\nabla\left(j_{l}^{h} p-p\right)\right\|_{\Omega_{D}}\left\|\mathbf{w}_{h}\right\|_{\Omega_{D}} \\
& \left.+\left\|\nabla q_{h}\right\|_{\Omega_{D}}\left\|\mathbf{j}_{r}^{h} \mathbf{v}-\mathbf{v}\right\|_{\Omega_{D}}\right] \\
& \leq \widetilde{\beta}^{-1} c_{i} \widetilde{C}\left[k_{2} h^{r+1}\|\mathbf{v}\|_{r+1, \Omega_{D}}+\delta h^{r}\|\mathbf{v}\|_{r+1, \Omega_{D}}\right. \\
& \left.+h^{l}\|p\|_{l+1, \Omega_{D}}+h^{r+1}\|\mathbf{v}\|_{r+1, \Omega_{D}}\right] \text {. }
\end{aligned}
$$

Thus,

$$
\begin{aligned}
\|\left(\mathbf{j}_{r}^{h} \mathbf{v}\right. & \left.-\mathbf{v}_{h}, j_{l}^{h} p-p_{h}\right) \|_{h} \\
\leq & \left(\widetilde{c}_{1} v+\widetilde{c}_{2}\right) h^{r}\|\mathbf{v}\|_{r+1, \Omega_{S}}+\left(\widetilde{c}_{3} h+\widetilde{c}_{4} \delta\right) h^{r}\|\mathbf{v}\|_{r+1, \Omega_{D}} \\
& +\left(\widetilde{c}_{5}+\widetilde{c}_{6} \gamma^{1 / 2} h^{1 / 2}+\widetilde{c}_{7} h\right) h^{l}\|p\|_{l+1, \Omega_{S}} \\
& +\left(\widetilde{c}_{5}+\widetilde{c}_{6} \gamma^{1 / 2} h^{1 / 2}+\widetilde{c}_{7} h\right) h^{l}\|p\|_{l+1, \Omega_{D}}
\end{aligned}
$$

Squaring the norm and applying Young inequality we obtain

$$
\begin{aligned}
\|\left(\mathbf{j}_{r}^{h} \mathbf{v}\right. & \left.-\mathbf{v}_{h}, j_{l}^{h} p-p_{h}\right) \|_{h}^{2} \\
\leq & 4\left(\widetilde{c}_{1} \nu+\widetilde{c}_{2}\right)^{2} h^{2 r}\|\mathbf{v}\|_{r+1, \Omega_{S}}^{2} \\
& +4\left(\widetilde{c}_{3} h+\widetilde{c}_{4} \delta\right)^{2} h^{2 r}\|\mathbf{v}\|_{r+1, \Omega_{D}}^{2} \\
& +4\left(\widetilde{c}_{5}+\widetilde{c}_{6} \gamma^{1 / 2} h^{1 / 2}+\widetilde{c}_{7} h\right)^{2} h^{2 l}\|p\|_{l+1, \Omega_{S}}^{2} \\
& +4\left(\widetilde{c}_{5}+\widetilde{c}_{6} \gamma^{1 / 2} h^{1 / 2}+\widetilde{c}_{7} h\right)^{2} h^{2 l}\|p\|_{l+1, \Omega_{D}}^{2} .
\end{aligned}
$$

Next, we estimate the interpolation error by

$$
\begin{aligned}
\left\|\left(\mathbf{v}-\mathbf{j}_{r}^{h} \mathbf{v}, p-j_{l}^{h} p\right)\right\|_{h}^{2} \\
=\left\|\nabla\left(\mathbf{v}-\mathbf{j}_{r}^{h} \mathbf{v}\right)\right\|_{\Omega_{S}}^{2}+\left\|\left(\mathbf{v}-\mathbf{j}_{r}^{h} \mathbf{v}\right)\right\|_{\Omega_{D}}^{2} \\
\quad+\left\|\operatorname{div}\left(\mathbf{v}-\mathbf{j}_{r}^{h} \mathbf{v}\right)\right\|_{\Omega_{D}}^{2}+\left\|p-j_{l}^{h} p\right\|_{\Omega_{S}}^{2} \\
\quad+\left\|\nabla\left(p-j_{l}^{h} p\right)\right\|_{\Omega_{D}}^{2}+\mathcal{S}_{h}\left(\kappa_{2 h} p, \kappa_{2 h} p\right) \\
\leq c_{i}^{2} h^{2 r}\|\mathbf{v}\|_{r+1, \Omega_{S}}^{2}+c_{i}^{2} h^{2 r}\left(h^{2}+1\right) h^{2 r}\|\mathbf{v}\|_{r+1, \Omega_{D}}^{2} \\
\quad+\left(\widetilde{c}_{i}^{2} h^{2}+\gamma h\right) h^{2 l}\|p\|_{l+1, \Omega_{S}}^{2} \\
\quad+\left(\widetilde{c}_{i}^{2}+\gamma h\right) h^{2 l}\|p\|_{l+1, \Omega_{D}}^{2} .
\end{aligned}
$$

Adding the interpolation error (64) to the projection error (65) we obtain the required result

$$
\begin{aligned}
\| & \left(\mathbf{v}-\mathbf{v}_{h}, p-p_{h}\right) \|_{h} \leq\left\{\left(c_{1} v+c_{2}\right)^{2} h^{2 r}\|\mathbf{v}\|_{r+1, \Omega_{S}}^{2}\right. \\
& +\left(c_{3} h+c_{4} \delta\right)^{2} h^{2 r}\|\mathbf{v}\|_{r+1, \Omega_{D}}^{2} \\
& +\left(c_{5}+c_{6} \gamma^{1 / 2} h^{1 / 2}+c_{7} h\right)^{2} h^{2 l}\|p\|_{l+1, \Omega_{S}}^{2} \\
& \left.+\left(c_{5}+c_{6} \gamma^{1 / 2} h^{1 / 2}+c_{7} h\right)^{2} h^{2 l}\|p\|_{l+1, \Omega_{D}}^{2}\right\}^{1 / 2} .
\end{aligned}
$$

Remark 4. We note that the analysis above holds true for the triangular subgrid interpolation $P_{r}-P_{r}-P_{r}$.

Remark 5. Because of the presence of divergence of the velocity and the gradient of the pressure in the discrete norm, the velocity and pressure solutions are $O\left(h^{r}\right)$ and $O\left(h^{l}\right)$, respectively. So, we expect the $L_{2}$-asymptotic rates to be $\mathrm{O}\left(h^{r+1}\right)$ and $\mathrm{O}\left(h^{l+1}\right)$.

\section{Numerical Results}

As a test model problem we take $\Omega=(0,1) \times(0,1)$ and split it into $\Omega_{S}=(0,1 / 2) \times(0,1)$ and $\Omega_{D}=(1 / 2,1) \times(0,1)$. The interface boundary is $\Gamma=\{(0.5, y) \mid 0<y<1\}$. We take 
TABLE 1: Rates of convergence for velocity and pressure solution in the Stokes subdomain.

\begin{tabular}{lccc}
\hline & $\left\|\mathbf{u}-\mathbf{u}_{h}\right\|_{0, \Omega_{S}}$ & $\left\|\nabla\left(\mathbf{u}-\mathbf{u}_{h}\right)\right\|_{0, \Omega_{S}}$ & $\left\|p-p_{h}\right\|_{0, \Omega_{S}}$ \\
\hline$h=\frac{1}{8}$ & - & - & - \\
$h=\frac{1}{16}$ & 1.9303 & 1.0284 & 0.8480 \\
$h=\frac{1}{32}$ & 1.9735 & 1.0208 & 0.9149 \\
$h=\frac{1}{64}$ & 1.9890 & 1.0119 & 0.9511 \\
$h=\frac{1}{128}$ & 1.9951 & 1.0055 & 0.9725 \\
\hline
\end{tabular}

TABLE 2: Rates of convergence for velocity and pressure solution in the Darcy subdomain.

\begin{tabular}{cccc}
\hline & $\left\|\mathbf{u}-\mathbf{u}_{h}\right\|_{0, \Omega_{D}}$ & $\left\|\operatorname{div}\left(\mathbf{u}-\mathbf{u}_{h}\right)\right\|_{0, \Omega_{D}}$ & $\left\|p-p_{h}\right\|_{0, \Omega_{D}}$ \\
\hline$h=\frac{1}{8}$ & - & - & - \\
$h=\frac{1}{16}$ & 0.8813 & 0.8412 & 1.0416 \\
$h=\frac{1}{32}$ & 0.9534 & 0.9235 & 1.0318 \\
$h=\frac{1}{64}$ & 0.9642 & 0.9514 & 1.0167 \\
$h=\frac{1}{128}$ & 0.9857 & 0.9657 & 1.0085 \\
\hline
\end{tabular}

$v=1, \alpha=1, \widetilde{k}=1$, and $K=I$ and the right hand sides f, $g$ such that the velocity and pressure solution in the two subdomains are given by

$$
\begin{aligned}
\mathbf{u}_{S} & =\left(y^{4} e^{x}, e^{y} \cos (2 x)\right), \quad(x, y) \in \Omega_{S} \\
\mathbf{u}_{D} & =\left(y^{4} e^{x}, 4 y^{3} e^{x}\right), \quad(x, y) \in \Omega_{D} \\
p & =y^{4} e^{x}, \quad(x, y) \in \Omega .
\end{aligned}
$$

Note that for this problem forcing terms are needed to balance the equations; notably additional terms are added to the interface conditions in (6) and (7) as follows:

$$
\begin{aligned}
&-\left(-p_{S} I+2 \nu D\left(\mathbf{v}_{S}\right)\right) \mathbf{n}_{S} \cdot \mathbf{n}_{S}=p_{D}+g_{1}, \quad \text { on } \Gamma, \\
& \mathbf{v}_{S} \cdot \tau=-\frac{2 \sqrt{\tilde{k}}}{\alpha}\left(D\left(\mathbf{v}_{S}\right) \cdot \mathbf{n}_{S}\right) \cdot \tau \\
& \text { on } \Gamma,
\end{aligned}
$$

where $g_{1}=-2 y^{4} e^{x}$, and $g_{2}=e^{y} \cos (2 x)+4 y^{3} e^{x}-2 e^{y} \sin (2 x)$.

The problem is solved using a $Q_{1}-Q_{1}$ velocity-pressure approximation with a two-level subgrid stabilization on a uniform mesh with $\delta=0.4$. Rates of convergence for the velocity and pressure errors for $h=1 / 8,1 / 16,1 / 32,1 / 64$, and $1 / 128$ are displayed in Tables 1 and 2 .

In Table 1, we see clearly that the velocity field in the Stokes subdomain is of second-order accuracy with respect to the $L_{2}$-norm and first-order accuracy with respect to $H^{1}$-seminorm, and the pressure is of first-order accuracy.
In addition, In Table 2, we observe that the velocity field and its divergence are of first-order accuracy in the Darcy subdomain, and the pressure is of first-order accuracy with respect to the $L_{2}$-norm. So, clearly these results are in agreement with the theoretical results of the previous section and are comparable to the ones found in $[2,5]$.

\section{Competing Interests}

The author declares that they have no competing interests.

\section{Acknowledgments}

The author acknowledges the financial support of the Sultan Qaboos University, under Contract IG/SCI/DOMS/14/07.

\section{References}

[1] W. J. Layton, F. Schieweck, and I. Yotov, "Coupling fluid flow with porous media flow," SIAM Journal on Numerical Analysis, vol. 40, no. 6, pp. 2195-2218, 2003.

[2] J. M. Urquiza, D. N’Dri, A. Garon, and M. C. Delfour, "Coupling Stokes and Darcy equations," Applied Numerical Mathematics, vol. 58, no. 5, pp. 525-538, 2008.

[3] V. Girault and B. Rivière, "DG approximation of coupled Navier-Stokes and Darcy equations by Beaver-Joseph-Saffman interface condition," SIAM Journal on Numerical Analysis, vol. 47, no. 3, pp. 2052-2089, 2009.

[4] T. Karper, K.-A. Mardal, and R. Winther, "Unified finite element discretizations of coupled Darcy-Stokes flow," Numerical Methods for Partial Differential Equations, vol. 25, no. 2, pp. 311-326, 2009.

[5] G. Pacquaut, J. Bruchon, N. Moulin, and S. Drapier, "Combining a level-set method and a mixed stabilized P1/P1 formulation for coupling Stokes-Darcy flows," International Journal for Numerical Methods in Fluids, vol. 69, no. 2, pp. 459-480, 2012.

[6] P. G. Saffman, "On the boundary condition at the surface of a porous medium," Studies in Applied Mathematics, vol. 50, no. 2, pp. 93-101, 1971.

[7] M. Braack and K. Nafa, "A uniform local projection finite element method for coupled Darcy-Stokes flow," in Proceedings of the 5th International Conference on Approximation Methods and Numerical Modeling in Environment and Natural Resources (MAMERN '13), Granada, Spain, April 2013.

[8] A. Mikelic and W. Jager, "On the interface boundary condition of Beavers, Joseph, and Saffman," SIAM Journal on Applied Mathematics (SIAP), vol. 60, no. 4, pp. 1111-1127, 2000.

[9] W. Wang and C. Xu, "Spectral methods based on new formulations for coupled Stokes and Darcy equations," Journal of Computational Physics, vol. 257, pp. 126-142, 2014.

[10] K. Nafa, "Equal order approximations enriched with bubbles for coupled Stokes-Darcy problem," Journal of Computational and Applied Mathematics, vol. 270, pp. 275-282, 2014.

[11] F. Brezzi and M. Fortin, Mixed and Hybrid Finite Element Methods, vol. 15 of Springer Series in Computational Mathematics, Springer, New York, NY, USA, 1991.

[12] A. Ern and J.-L. Guermond, Theory and Practice of Finite Elements, vol. 159 of Applied Mathematical Sciences, Springer, New York, NY, USA, 2004. 
[13] S. C. Brenner and L. R. Scott, The Mathematical Theory of Finite Element Methods, vol. 15 of Texts in Applied Mathematics, Springer, New York, NY, USA, 3rd edition, 2008.

[14] R. Li, J. Li, Z. Chen, and Y. Gao, "A stabilized finite element method based on two local Gauss integrations for a coupled Stokes-Darcy problem," Journal of Computational and Applied Mathematics, vol. 292, pp. 92-104, 2016.

[15] J. L. Guermond, "Stabilization of Galerkin approximations of monotone operators," IMA Journal of Numerical Analysis, vol. 21, pp. 165-197, 2001.

[16] S. Badia and R. Codina, "Unified stabilized finite element formulations for the Stokes and the Darcy problems," SIAM Journal on Numerical Analysis, vol. 47, no. 3, pp. 1971-2000, 2009. 


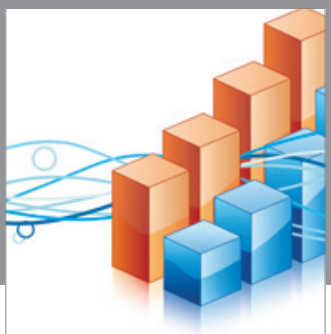

Advances in

Operations Research

vatem alat4

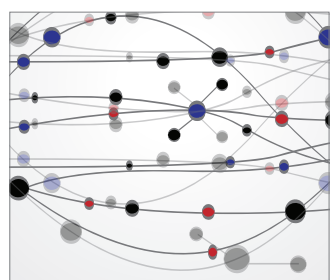

\section{The Scientific} World Journal
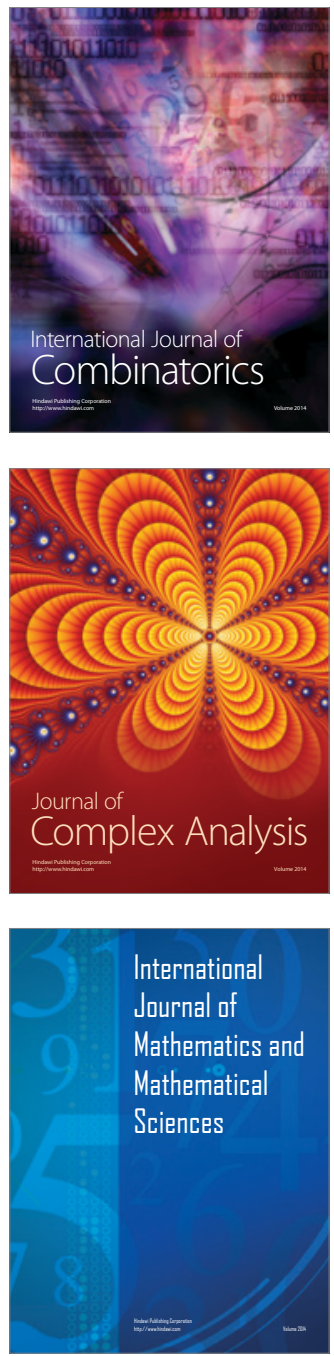
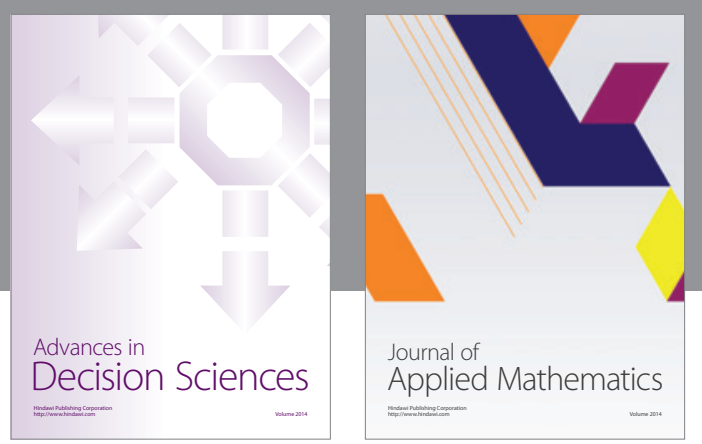

Algebra

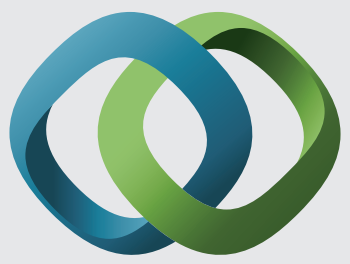

\section{Hindawi}

Submit your manuscripts at

http://www.hindawi.com
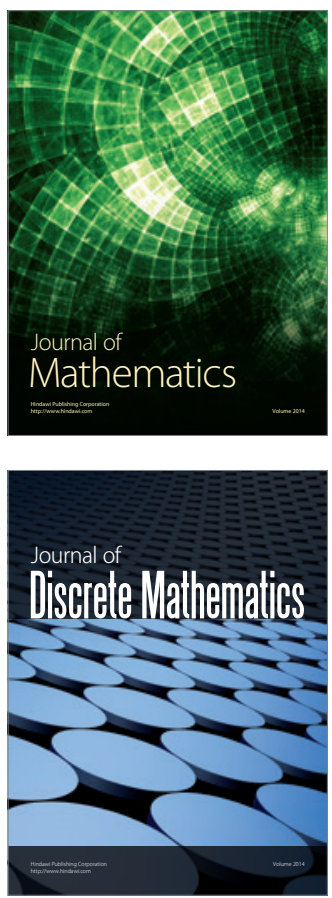

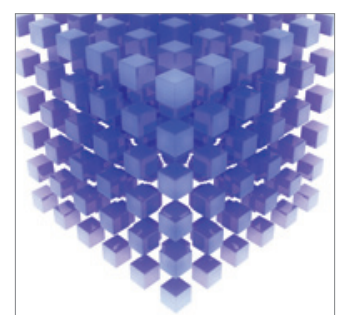

Mathematical Problems in Engineering
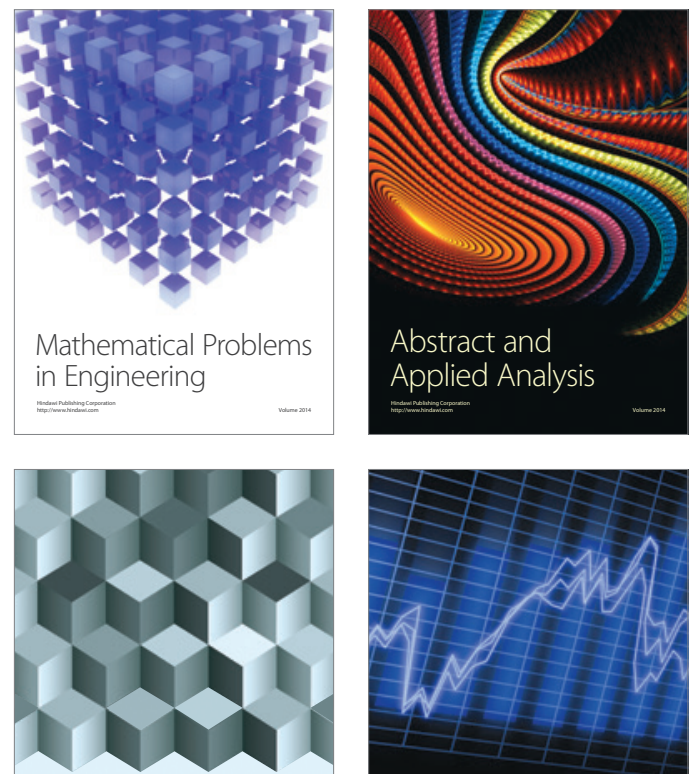

Journal of

Function Spaces

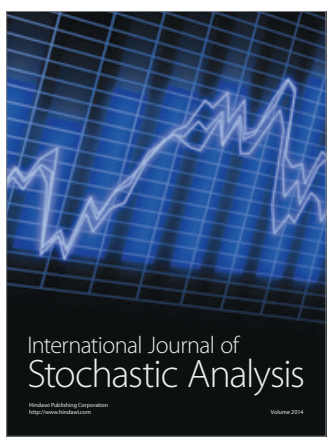

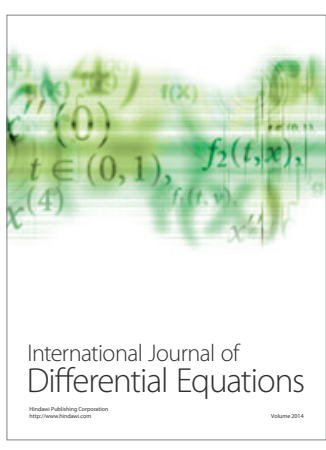
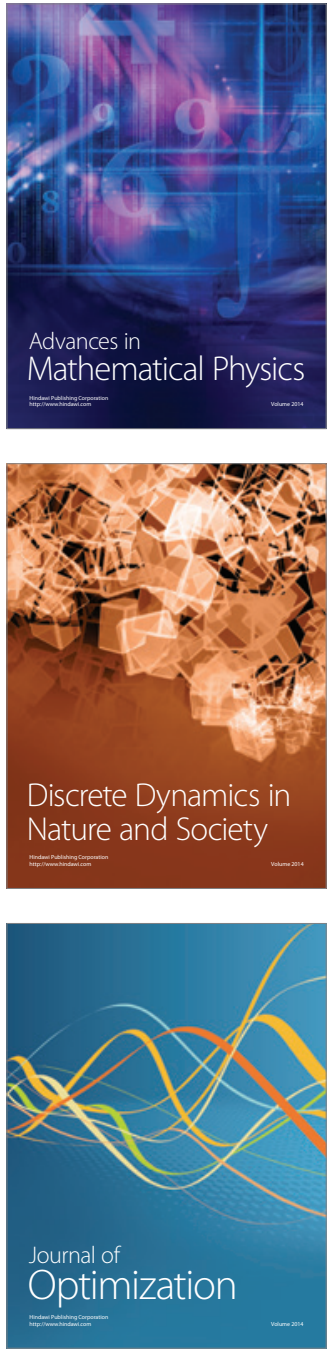